\title{
Uniqueness of Factoring an Integer and Multiplicative Group $\mathbb{Z} / p \mathbb{Z}^{*}$
}

\author{
Hiroyuki Okazaki \\ Shinshu University \\ Nagano, Japan
}

\author{
Yasunari Shidama \\ Shinshu University \\ Nagano, Japan
}

\begin{abstract}
Summary. In the [20], it had been proven that the Integers modulo $p$, in this article we shall refer as $\mathbb{Z} / p \mathbb{Z}$, constitutes a field if and only if $p$ is a prime. Then the prime modulo $\mathbb{Z} / p \mathbb{Z}$ is an additive cyclic group and $\mathbb{Z} / p \mathbb{Z}^{*}=\mathbb{Z} / p \mathbb{Z} \backslash\{0\}$ is a multiplicative cyclic group, too. The former has been proven in the [23]. However, the latter had not been proven yet. In this article, first, we prove a theorem concerning the LCM to prove the existence of primitive elements of $\mathbb{Z} / p^{*}$. Moreover we prove the uniqueness of factoring an integer. Next we define the multiplicative group $\mathbb{Z} / p \mathbb{Z}^{*}$ and prove it is cyclic.
\end{abstract}

MML identifier: INT_7 , version: $\underline{7.8 .10 \quad 4.99 .1005}$

The articles [31], [3], [9], [1], [25], [2], [32], [8], [24], [4], [19], [29], [28], [13], [7], [26], [22], [11], [17], [18], [12], [16], [30], [23], [27], [5], [14], [15], [20], [21], [6], and [10] provide the terminology and notation for this paper.

\section{Uniqueness of FACTORING AN Integer}

In this paper $x, X$ denote sets.

Next we state four propositions:

(1) For every many sorted set $p$ indexed by $X$ such that support $p=\{x\}$ holds $p=(X \longmapsto 0)+\cdot(x, p(x))$.

(2) Let $X$ be a set and $p, q, r$ be real-valued many sorted sets indexed by $X$. If support $p \cap \operatorname{support} q=\emptyset$ and $\operatorname{support} p \cup \operatorname{support} q=\operatorname{support} r$ and $p\lceil\operatorname{support} p=r\lceil\operatorname{support} p$ and $q\lceil\operatorname{support} q=r\lceil$ support $q$, then $p+q=r$. 
(3) For every set $X$ and for all many sorted sets $p, q$ indexed by $X$ such that $p\lceil\operatorname{support} p=q\lceil\operatorname{support} q$ holds $p=q$.

(4) For every set $X$ and for all bags $p, q$ of $X$ such that support $p=\emptyset$ and support $q=\emptyset$ holds $p=q$. if:

Let $p$ be a bag of Prime. We say that $p$ is prime-factorization-like if and only

(Def. 1) For every prime number $x$ such that $x \in \operatorname{support} p$ there exists a natural number $n$ such that $0<n$ and $p(x)=x^{n}$.

Let $n$ be a non empty natural number. Note that $\operatorname{PPF}(n)$ is prime-factorizationlike.

Next we state a number of propositions:

(5) For all prime numbers $p, q$ and for all natural numbers $n, m$ such that $p \mid m \cdot q^{n}$ and $p \neq q$ holds $p \mid m$.

(6) Let $f$ be a finite sequence of elements of $\mathbb{N}, b$ be a bag of Prime, and $a$ be a prime number. Suppose $b$ is prime-factorization-like and $\prod b \neq 1$ and $a \mid \prod b$ and $\prod b=\prod f$ and $f=b \cdot \operatorname{CFS}(\operatorname{support} b$ ). Then $a \in \operatorname{support} b$.

(7) For all bags $p, q$ of Prime such that support $p \subseteq \operatorname{support} q$ and $p\left\lceil\operatorname{support} p=q\left\lceil\operatorname{support} p\right.\right.$ holds $\prod p \mid \prod q$.

(8) Let $p$ be a bag of Prime and $x$ be a prime number. If $p$ is primefactorization-like, then $x \mid \prod p$ iff $x \in \operatorname{support} p$.

(9) For all non empty natural numbers $n, m, k$ such that $k=\operatorname{lcm}(n, m)$ holds support $\operatorname{PPF}(k)=\operatorname{support} \operatorname{PPF}(n) \cup \operatorname{support} \operatorname{PPF}(m)$.

(10) For every set $X$ and for all bags $b_{1}, b_{2}$ of $X$ holds support $\min \left(b_{1}, b_{2}\right)=$ support $b_{1} \cap \operatorname{support} b_{2}$.

(11) For all non empty natural numbers $n, m, k$ such that $k=n \operatorname{gcd} m$ holds $\operatorname{support} \operatorname{PPF}(k)=\operatorname{support} \operatorname{PPF}(n) \cap \operatorname{support} \operatorname{PPF}(m)$.

(12) Let $p, q$ be bags of Prime. Suppose $p$ is prime-factorization-like and $q$ is prime-factorization-like and support $p$ misses support $q$. Then $\prod p$ and $\prod q$ are relative prime.

(13) For every bag $p$ of Prime such that $p$ is prime-factorization-like holds $\prod p \neq 0$.

(14) For every bag $p$ of Prime such that $p$ is prime-factorization-like holds $\prod p=1$ iff $\operatorname{support} p=\emptyset$.

(15) Let $p, q$ be bags of Prime. Suppose $p$ is prime-factorization-like and $q$ is prime-factorization-like and $\prod p=\prod q$. Then $p=q$.

(16) Let $p$ be a bag of Prime and $n$ be a non empty natural number. If $p$ is prime-factorization-like and $n=\prod p$, then $\operatorname{PPF}(n)=p$.

(17) Let $n, m$ be elements of $\mathbb{N}$. Suppose $1 \leq n$ and $1 \leq m$. Then there exist elements $m_{0}, n_{0}$ of $\mathbb{N} \operatorname{such}$ that $\operatorname{lcm}(n, m)=n_{0} \cdot m_{0}$ and $n_{0} \operatorname{gcd} m_{0}=1$ 
and $n_{0} \mid n$ and $m_{0} \mid m$ and $n_{0} \neq 0$ and $m_{0} \neq 0$.

\section{Multiplicative Group $\mathbb{Z} / p \mathbb{Z}^{*}$}

Let $n$ be a natural number. Let us assume that $1<n$. The functor $\mathbb{Z}_{n}^{*}$ yields a non empty finite subset of $\mathbb{N}$ and is defined by:

(Def. 2) $\mathbb{Z}_{n}^{*}=\mathbb{Z}_{n} \backslash\{0\}$.

We now state the proposition

(18) For every natural number $n$ such that $1<n$ holds $\overline{\overline{\mathbb{Z}_{n}^{*}}}=n-1$.

Let $n$ be a prime number. The functor $\bullet_{\mathbb{Z}_{n}^{*}}$ yielding a binary operation on $\mathbb{Z}_{n}^{*}$ is defined by:

(Def. 3) $\quad \cdot \mathbb{Z}_{n}^{*}=\cdot_{\mathbb{Z}_{n}} \uparrow \mathbb{Z}_{n}^{*}$.

One can prove the following proposition

(19) For every prime number $p$ holds $\left\langle\mathbb{Z}_{p}^{*}, \cdot_{p}^{*}\right\rangle$ is associative, commutative, and group-like.

Let $p$ be a prime number. The functor $\mathbb{Z} / p \mathbb{Z}^{*}$ yielding a commutative group is defined by:

(Def. 4) $\mathbb{Z} / p \mathbb{Z}^{*}=\left\langle\mathbb{Z}_{p}^{*}, \mathbb{Z}_{p}^{*}\right\rangle$.

The following three propositions are true:

(20) Let $p$ be a prime number, $x, y$ be elements of $\mathbb{Z} / p \mathbb{Z}^{*}$, and $x_{1}, y_{1}$ be elements of $\mathbb{Z}_{p}^{\mathrm{R}}$. If $x=x_{1}$ and $y=y_{1}$, then $x \cdot y=x_{1} \cdot y_{1}$.

(21) For every prime number $p$ holds $\mathbf{1}_{\mathbb{Z} / p \mathbb{Z}^{*}}=1$ and $\mathbf{1}_{\mathbb{Z} / p \mathbb{Z}^{*}}=1_{\mathbb{Z}_{p}^{\mathrm{R}}}$.

(22) For every prime number $p$ and for every element $x$ of $\mathbb{Z} / p \mathbb{Z}^{*}$ and for every element $x_{1}$ of $\mathbb{Z}_{p}^{\mathrm{R}}$ such that $x=x_{1}$ holds $x^{-1}=x_{1}{ }^{-1}$.

Let $p$ be a prime number. One can verify that $\mathbb{Z} / p \mathbb{Z}^{*}$ is finite.

We now state several propositions:

(23) For every prime number $p$ holds $\operatorname{ord}\left(\mathbb{Z} / p \mathbb{Z}^{*}\right)=p-1$.

(24) Let $G$ be a group, $a$ be an element of $G$, and $i$ be an integer. Suppose $a$ is not of order 0 . Then there exist elements $n, k$ of $\mathbb{N}$ such that $a^{i}=a^{n}$ and $n=k \cdot \operatorname{ord}(a)+i$.

(25) Let $G$ be a commutative group, $a, b$ be elements of $G$, and $n, m$ be natural numbers. If $G$ is finite and $\operatorname{ord}(a)=n$ and $\operatorname{ord}(b)=m$ and $n \operatorname{gcd} m=1$, then $\operatorname{ord}(a \cdot b)=n \cdot m$.

(26) For every non empty zero structure $L$ and for every polynomial $p$ of $L$ such that $0 \leq \operatorname{deg} p$ holds $p$ is non-zero.

(27) For every field $L$ and for every polynomial $f$ of $L$ such that $0 \leq \operatorname{deg} f$ holds Roots $f$ is a finite set and $\overline{\overline{\operatorname{Roots} f}} \leq \operatorname{deg} f$. 
(28) Let $p$ be a prime number, $z$ be an element of $\mathbb{Z} / p \mathbb{Z}^{*}$, and $y$ be an element of $\mathbb{Z}_{p}^{\mathrm{R}}$. If $z=y$, then for every element $n$ of $\mathbb{N}$ holds $\operatorname{power}_{\mathbb{Z} / p \mathbb{Z}^{*}}(z, n)=$ $\operatorname{power}_{\mathbb{Z}_{p}^{\mathrm{R}}}(y, n)$.

(29) Let $p$ be a prime number, $a, b$ be elements of $\mathbb{Z} / p \mathbb{Z}^{*}$, and $n$ be a natural number. If $0<n$ and $\operatorname{ord}(a)=n$ and $b^{n}=1$, then $b$ is an element of $\operatorname{gr}(\{a\})$.

(30) Let $G$ be a group, $z$ be an element of $G$, and $d, l$ be elements of $\mathbb{N}$. If $G$ is finite and $\operatorname{ord}(z)=d \cdot l$, then $\operatorname{ord}\left(z^{d}\right)=l$.

(31) For every prime number $p$ holds $\mathbb{Z} / p \mathbb{Z}^{*}$ is a cyclic group.

\section{REFERENCES}

[1] Grzegorz Bancerek. Cardinal numbers. Formalized Mathematics, 1(2):377-382, 1990.

[2] Grzegorz Bancerek. The fundamental properties of natural numbers. Formalized Mathematics, 1(1):41-46, 1990.

[3] Grzegorz Bancerek. The ordinal numbers. Formalized Mathematics, 1(1):91-96, 1990.

[4] Grzegorz Bancerek and Krzysztof Hryniewiecki. Segments of natural numbers and finite sequences. Formalized Mathematics, 1(1):107-114, 1990.

[5] Grzegorz Bancerek and Andrzej Trybulec. Miscellaneous facts about functions. Formalized Mathematics, 5(4):485-492, 1996.

[6] Józef Białas. Group and field definitions. Formalized Mathematics, 1(3):433-439, 1990.

[7] Czesław Byliński. Binary operations. Formalized Mathematics, 1(1):175-180, 1990.

[8] Czesław Byliński. Functions and their basic properties. Formalized Mathematics, 1(1):5565, 1990.

[9] Agata Darmochwał. Finite sets. Formalized Mathematics, 1(1):165-167, 1990.

[10] Krzysztof Hryniewiecki. Recursive definitions. Formalized Mathematics, 1(2):321-328, 1990.

[11] Andrzej Kondracki. The Chinese Remainder Theorem. Formalized Mathematics, 6(4):573-577, 1997.

[12] Artur Korniłowicz and Piotr Rudnicki. Fundamental Theorem of Arithmetic. Formalized Mathematics, 12(2):179-186, 2004.

[13] Eugeniusz Kusak, Wojciech Leończuk, and Michał Muzalewski. Abelian groups, fields and vector spaces. Formalized Mathematics, 1(2):335-342, 1990.

[14] Rafał Kwiatek. Factorial and Newton coefficients. Formalized Mathematics, 1(5):887-890, 1990.

[15] Rafał Kwiatek and Grzegorz Zwara. The divisibility of integers and integer relative primes. Formalized Mathematics, 1(5):829-832, 1990.

[16] Robert Milewski. Fundamental theorem of algebra. Formalized Mathematics, 9(3):461470, 2001.

[17] Robert Milewski. The ring of polynomials. Formalized Mathematics, 9(2):339-346, 2001.

[18] Piotr Rudnicki. Little Bezout theorem (factor theorem). Formalized Mathematics, 12(1):49-58, 2004.

[19] Piotr Rudnicki and Andrzej Trybulec. Multivariate polynomials with arbitrary number of variables. Formalized Mathematics, 9(1):95-110, 2001.

[20] Christoph Schwarzweller. The ring of integers, euclidean rings and modulo integers. Formalized Mathematics, 8(1):29-34, 1999.

[21] Christoph Schwarzweller and Agnieszka Rowińska-Schwarzweller. Schur's theorem on the stability of networks. Formalized Mathematics, 14(4):135-142, 2006.

[22] Christoph Schwarzweller and Andrzej Trybulec. The evaluation of multivariate polynomials. Formalized Mathematics, 9(2):331-338, 2001.

[23] Dariusz Surowik. Cyclic groups and some of their properties - part I. Formalized Mathematics, 2(5):623-627, 1991.

[24] Andrzej Trybulec. Binary operations applied to functions. Formalized Mathematics, $1(2): 329-334,1990$. 
[25] Andrzej Trybulec. Domains and their Cartesian products. Formalized Mathematics, 1(1):115-122, 1990.

[26] Andrzej Trybulec. Many-sorted sets. Formalized Mathematics, 4(1):15-22, 1993.

[27] Michał J. Trybulec. Integers. Formalized Mathematics, 1(3):501-505, 1990.

[28] Wojciech A. Trybulec. Groups. Formalized Mathematics, 1(5):821-827, 1990.

[29] Wojciech A. Trybulec. Vectors in real linear space. Formalized Mathematics, 1(2):291-296, 1990.

[30] Wojciech A. Trybulec. Lattice of subgroups of a group. Frattini subgroup. Formalized Mathematics, 2(1):41-47, 1991.

[31] Zinaida Trybulec. Properties of subsets. Formalized Mathematics, 1(1):67-71, 1990.

[32] Edmund Woronowicz. Relations and their basic properties. Formalized Mathematics, 1(1):73-83, 1990.

Received January 31, 2008 